\title{
Asymptotic Behavior of the Steklov Eigenvalues For the $p$-Laplace Operator
}

\author{
Juan Pablo Pinasco* \\ Instituto de Ciencias, Universidad Nac. de Gral. Sarmiento \\ J.M. Gutierrez 1150 - Los Polvorines (1613), \\ Buenos Aires, Argentina \\ e-mail: jpinasco@dm.uba.ar \\ Received, October 26, 2007 \\ Communicated by Ireneo Peral
}

\begin{abstract}
In this paper we study the asymptotic behavior of the Steklov eigenvalues of the $p$ Laplacian. We show the existence of lower and upper bounds of a Weyl-type expansion of the function $N(\lambda)$ which counts the number of eigenvalues less than or equal to $\lambda$, and we derive from them asymptotic bounds for the eigenvalues.
\end{abstract}

1991 Mathematics Subject Classification. 35P20, 35P30.

Key words. p-laplacian, asymptotic of eigenvalues, Steklov

\section{Introduction}

In this paper we study the following eigenvalue problem:

$$
\left\{\begin{aligned}
\Delta_{p} u & =|u|^{p-2} u & & \text { in } \Omega \\
|\nabla u|^{p-2} \frac{\partial u}{\partial \nu} & =\lambda|u|^{p-2} u & & \text { on } \partial \Omega
\end{aligned}\right.
$$

in a bounded open set $\Omega \subset \mathbb{R}^{N}$ with smooth boundary $\partial \Omega$ (at least, $C^{2}$ ), where $\Delta_{p} u$ is the $p$-laplacian operator $\operatorname{div}\left(|\nabla u|^{p-2} \nabla u\right)$ for $1<p<+\infty, \partial / \partial \nu$ is the outer unit normal

*This work has been supported by UBA, UNGS, and ANPCyT PICT No. 03-05009. I wish to thank Drs. J. Fernández Bonder, P. de Nápoli and J. Rossi for many interesting conversations. 
derivative, and $\lambda$ is a real parameter. The case $p=+\infty$ will be considered in a separate work (see [6]).

From [7], we know that the variational spectrum consists on a countable sequence of positive eigenvalues $\lambda_{1}<\lambda_{2} \leq \ldots \leq \lambda_{k} \leq \ldots$ (repeated according multiplicity) tending to $+\infty$, which are obtained with the aid of the Ljusternik-Schnirelmann theory. We recall that the variational characterization of the eigenvalues is as follows:

$$
\lambda_{k}=\inf _{F \in C_{k}^{\Omega}} \sup _{u \in F} \int_{\Omega}|\nabla u|^{p}+|u|^{p},
$$

where

$$
\begin{gathered}
C_{k}^{\Omega}=\left\{C \subset M^{\Omega}: C \text { compact }, C=-C, \gamma(C) \geq k\right\}, \\
M^{\Omega}=\left\{u \in W^{1, p}(\Omega): \int_{\partial \Omega}|u|^{p}=1\right\}
\end{gathered}
$$

and $\gamma(C)$ is the Krasnoselskii genus (see [17] for the definition and properties of $\gamma$ ).

However, few facts are known about this sequence. It is not known if this variational sequence exhausts the spectrum, although it was proved that $\lambda_{1}$ is the only eigenvalue with a positive eigenfunction, it is simple, and there are no other eigenvalues between $\lambda_{1}$ and $\lambda_{2}$, see [15]. Moreover, it can be obtained by minimization of the Rayleigh quotient over all of $W^{1, p}(\Omega)$ :

$$
\lambda_{1}=\min _{u \in W^{1, p}(\Omega) \backslash 0} \frac{\int_{\Omega}|\nabla u|^{p}+|u|^{p}}{\int_{\partial \Omega}|u|^{p}} .
$$

Also, $\lambda_{2}$ is a variational eigenvalue which can be obtained by minimization in a different set of functions, and the corresponding eigenfunction changes signs (see [8] for details).

Our objective is to obtain the asymptotic growth of this sequence. To this end, we introduce the spectral counting function $N(\lambda)$ defined as the number of variational eigenvalues of problem (1.1) less than a given $\lambda$ :

$$
N(\lambda, \Omega)=\#\left\{k: \lambda_{k} \leq \lambda\right\} .
$$

We obtain two side estimates of the asymptotic expansion of $N(\lambda)$, bounding it above and by below. We state here our main result:

Theorem 1.1 Let $\left\{\lambda_{k}\right\}_{k}$ be the sequence of variational eigenvalues of Problem 1.1. Then,

$$
c|\partial \Omega|_{N-1} \lambda^{\frac{N-1}{p-1}} \leq N(\lambda) \leq C|\partial \Omega|_{N-1} \lambda^{\frac{N-1}{p-1}},
$$

for certain positive constants $c, C$ depending only on $p$ and $N$.

The proof is based on variational arguments, by using an extension of the 'DirichletNeumann bracketing' method of Courant, see [2]. As a corollary, we have:

Corollary 1.1 Let $\left\{\lambda_{k}\right\}_{k}$ be the sequence of variational eigenvalues of Problem 1.1. Then, there exists two positive constants $c_{1}$ and $c_{2}$ depending only on $p$ and $N$ such that

$$
c_{1}\left(\frac{k}{|\partial \Omega|_{N-1}}\right)^{\frac{p-1}{N-1}} \leq \lambda_{k} \leq c_{2}\left(\frac{k}{|\partial \Omega|_{N-1}}\right)^{\frac{p-1}{N-1}} .
$$


Let us emphasize the fact that $c_{1}$ and $c_{2}$ (and also $c, C$ in the previous theorem) are independent of the set $\Omega$.

The problem of estimating the spectral counting function of different elliptic operators has a long history in the linear case $p=2$, see for instance $[2,13,14]$ and the references therein for the Laplacian. The linear Steklov problem was studied by L. Sandgren in [18], and we will follow his method of proof. The main difference is due to our impossibility to compute the Steklov eigenvalues in a square explicitly.

The nonlinear case is by far more recent. Concerning the variational eigenvalues $\left\{\mu_{k}\right\}_{k}$ of the $p$-Laplacian with zero Dirichlet boundary conditions, the first result was obtained by Garcia Azorero and Peral Alonso in [12],

$$
c k^{p / N} \leq \mu_{k} \leq C k^{p / N},
$$

which was improved by Friedlander in [11]. The one dimensional case was refined in $[4,16]$ where the following stronger result was obteined:

$$
\lim _{k \rightarrow \infty} \frac{\mu_{k}}{k^{p}}=\frac{\pi_{p}^{p}}{|\Omega|^{p}},
$$

where $\pi_{p}$ is defined as $\pi_{p}=2(p-1)^{1 / p} \int_{0}^{1}\left(1-s^{p}\right)^{-1 / p} d s$ (see [3]).

The paper is organized as follows: In $\S 2$ we introduce several auxiliary results concerning the eigenvalue problem 1.1 together with different tools which will be used in the paper. In $\S 3$ we obtain an upper bound for $N(\lambda)$ in a cube $Q$ with the eigenvalue in one face and zero Neumann boundary conditions in the others. $\S 4$ is devoted to the upper bound for $N(\lambda)$ in a rectangle $R$ with the eigenvalue in one face and zero Dirichlet boundary conditions in the others. Finally, in $\S 5$ we prove Theorem 1.1.

\section{Preliminaries}

The main tool in the following sections is the Dirichlet Neumann Bracketing introduced by Courant in [2] and extended to the nonlinear setting in [11] and independently in [4]. For the Steklov problem it was extended in [18], and we will only sketch it for our case.

We introduce first certain spaces and eigenvalue problems. Given an open set $\Omega \subset \mathbb{R}^{N}$, let $\partial \Omega=\overline{\Gamma_{1} \cup \Gamma_{2}}$, where $\Gamma_{1}$ and $\Gamma_{2}$ are relative open sets of $\partial \Omega$; and now we have the following problems:

Semi-Dirichlet Steklov eigenvalue problem: Find $\Lambda \in \mathbb{R}$ and $u \in W_{0, \Gamma_{2}}^{1, p}(\Omega)$ such that

$$
\left\{\begin{aligned}
\Delta_{p} u & =|u|^{p-2} u & & \text { in } \Omega \\
|\nabla u|^{p-2} \frac{\partial u}{\partial \nu} & =\Lambda|u|^{p-2} u & & \text { on } \Gamma_{1} \\
u & =0 & & \text { on } \Gamma_{2} .
\end{aligned}\right.
$$

Semi-Neumann Steklov eigenvalue problem: Find $\Lambda \in \mathbb{R}$ and $u \in W^{1, p}(\Omega)$ such that

$$
\left\{\begin{aligned}
\Delta_{p} u & =|u|^{p-2} u & & \text { in } \Omega \\
|\nabla u|^{p-2} \frac{\partial u}{\partial \nu} & =\Lambda|u|^{p-2} u & & \text { on } \Gamma_{1} \\
\frac{\partial u}{\partial \nu} & =0 & & \text { on } \Gamma_{2} .
\end{aligned}\right.
$$


For both problems there exists a sequence of variational eigenvalues given by a characterization similar to 1.2 , changing only the definition of $M^{\Omega}$ as

$$
M^{\Omega}=\left\{u \in W_{0, \Gamma_{2}}^{1, p}(\Omega): \int_{\Gamma_{1}}|u|^{p}=1\right\}
$$

for the semi-Dirichlet problem, and

$$
M^{\Omega}=\left\{u \in W^{1, p}(\Omega): \int_{\Gamma_{1}}|u|^{p}=1\right\}
$$

for the semi-Neumann problem.

Also, it is not difficult to show that for both problems the first eigenvalue is positive and the associate eigenfunction is nonnegative. We leave the details for the reader, although they can be grasped from [7] and [15].

The asymptotic number of Steklov eigenvalues of problem 1.1 will be obtained from the spectral asymptotic of both problems, and we will write $N_{s D}\left(\lambda, \Omega, \Gamma_{1}\right), N_{s N}\left(\lambda, \Omega, \Gamma_{1}\right)$ to denote their counting functions. To this end, we introduce the Dirichlet-Neumann bracketing. We consider the Steklov problem 1.1 in $\Omega$, and we divide it in two disjoint open sets $\Omega_{1}, \Omega_{2} \in \mathbb{R}^{N}$ such that $\left(\overline{\Omega_{1} \cup \Omega_{2}}\right)^{\text {int }}=\Omega$ and $\left|\Omega \backslash \Omega_{1} \cup \Omega_{2}\right|_{N}=0$. Let $\Gamma_{2}$ be the common boundary $\overline{\Omega_{1}} \cap \overline{\Omega_{2}}$, and $\Gamma_{1, i}=\overline{\Omega_{i}} \cap \partial \Omega$ for $1 \leq i \leq 2$.

For such decomposition we have the following inequalities

$$
N_{s D}\left(\lambda, \Omega_{1}, \Gamma_{1,1}\right)+N_{s D}\left(\lambda, \Omega_{2}, \Gamma_{1,2}\right) \leq N(\lambda, \Omega)
$$

and

$$
N(\lambda, \Omega) \leq N_{s N}\left(\lambda, \Omega_{1}, \Gamma_{1,1}\right)+N_{s N}\left(\lambda, \Omega_{2}, \Gamma_{1,2}\right),
$$

which give the asymptotic behavior for problem 1.1 in terms of the other problems.

The proof of this fact is a consequence of the variational characterization of the eigenvalues 1.2 combined with the following inclusions:

$$
W_{0, \Gamma_{2}}^{1, p}\left(\Omega_{1} \cup \Omega_{2}\right)=W_{0, \Gamma_{2}}^{1, p}\left(\Omega_{1}\right) \oplus W_{0, \Gamma_{2}}^{1, p}\left(\Omega_{2}\right) \subset W^{1, p}(\Omega)
$$

and

$$
W^{1, p}(\Omega) \subset W^{1, p}\left(\Omega_{1}\right) \oplus W^{1, p}\left(\Omega_{2}\right)=W^{1, p}\left(\Omega_{1} \cup \Omega_{2}\right) .
$$

Let us note that, since $\Omega_{1}$ and $\Omega_{2}$ are disjoint sets, the eigenvalues are the union of the eigenvalues of the problem in each set separately.

Also, we will need some auxiliary results for problems with the eigenvalue parameter in the equation.

Let us consider following mixed eigenvalue problem:

$$
\left\{\begin{aligned}
-\Delta_{p} u & =\mu|u|^{p-2} u & & \text { in } R_{L} \\
\frac{\partial u}{\partial \nu} & =0 & & \text { on } \Gamma \\
u & =0 & & \text { on } \partial R_{L} \backslash \Gamma
\end{aligned}\right.
$$

where $R_{L}=[0, L]^{N-1} \times[0, L / 2]$, and $\Gamma$ is the face $\left\{\left(x_{1}, \ldots, x_{N}\right): 0 \leq x_{i} \leq L\right.$ for $1 \leq$ $i \leq N-1 ;$ and $\left.x_{N}=L / 2\right\}$. 
By a symmetry argument, the first eigenvalue $\mu_{1}$ coincides with the first Dirichlet eigenvalue corresponding to the cube $Q_{L}$. Indeed, by extending the first eigenfunction $u$ outside $R_{L}$ as $u\left(x_{1}, \ldots, x_{N-1}, L / 2+x\right)=u\left(x_{1}, \ldots, x_{N-1}, L / 2-x\right)$ for $x \in(0, L / 2)$, we obtain a Dirichlet eigenfunction, since it is a weak solution of problem 1.1, and being positive, it must be the first eigenfunction. Let us note also that the maximum of the function is attained at the center of $Q_{L}$.

We have the following result, which can be found also in [5]:

Proposition 2.1 Let $\mu_{1}^{\left(R_{L}\right)}$ be the first eigenvalue of Problem 2.7. Then,

$$
\mu_{1}^{\left(R_{L}\right)} \leq \begin{cases}\frac{\pi_{p}^{p} N^{p / 2}}{\pi_{p}^{L^{p}}} & \text { if } 2<p \\ \frac{\pi^{p}}{L^{p}} & \text { if } p<2 .\end{cases}
$$

Proof. In order to get a bound for $\mu_{1}^{\left(R_{L}\right)}$, we will study a different operator, the so-called pseudo $p$-Laplacian on $Q_{L}$ :

$$
\begin{cases}-\sum_{i=1}^{N} \frac{\partial}{\partial x_{i}}\left(\left|\frac{\partial v}{\partial x_{i}}\right|^{p-2} \frac{\partial v}{\partial x_{i}}\right)=\nu|v|^{p-2} v & \text { in } Q_{L} \\ v=0 & \text { in } \partial Q_{L} .\end{cases}
$$

Due to the equivalence of norms in $\mathbb{R}^{N}$, we have $|\nabla u|_{q} \leq C_{p}|\nabla u|_{p}$, where $C_{p}=1$ if $p \leq q$, and $C_{p}=N^{(p-q) / 2 q}$ if $p \geq q$ (see, for instance, [10]).

Since $\nu_{1}=\inf _{u \in W_{0}^{1, p}} \frac{\int_{Q_{L}} \sum_{i=1}^{N}\left|\frac{\partial u}{\partial x_{i}}\right|^{p}}{\int_{Q_{L}}|u|^{p}}$, by fixing the set $B=\left\{u \in W_{0}^{1, p}: \int_{Q_{L}}|u|^{p}\right\}$, we have the following characterization of the first eigenvalues $\mu_{1}, \nu_{1}$ of the $p$-laplacian and the pseudo $p$-Laplacian in $Q_{L} \subset \mathbb{R}^{N}$ respectively:

$$
\nu_{1}=\inf _{u \in B}\left\||\nabla u|_{p}\right\|_{p}^{p} ; \quad \mu_{1}=\inf _{u \in B}\left\||\nabla u|_{2}\right\|_{p}^{p},
$$

and the norms inequality enable us to compare $\nu_{1}$ and $\mu_{1}$.

Now, we have an explicit expression for $\nu_{1}$ :

$$
v_{1}=\sin _{p}\left(\pi_{p} x_{1} / L\right) \cdots \sin _{p}\left(\pi_{p} x_{N} / L\right), \quad \nu_{1}=\frac{\pi_{p}^{p} N}{L^{p}}
$$

is the first eigenpair of the pseudo $p$-Laplacian on $Q_{L}$. This result follows directly by separation of variables, and $v_{1}$ is the first eigenfunction since there exists only one positive eigenfunction of the pseudo $p$-laplacian (see [1]).

\section{An Upper Bound for $N(\lambda)$}

We will study the asymptotic behavior of the eigenvalues of the following semi-Neumann problem:

$$
\left\{\begin{aligned}
\Delta_{p} u & =|u|^{p-2} u & & \text { in } \Omega \\
|\nabla u|^{p-2} \frac{\partial u}{\partial \nu} & =\Lambda|u|^{p-2} u & & \text { on } \Gamma \\
\frac{\partial u}{\partial \nu} & =0 & & \text { on } \partial \Omega \backslash \Gamma .
\end{aligned}\right.
$$


Let us consider first the particular case $\Omega=Q_{L} \subset \mathbb{R}^{N}$, the cube $[0, L]^{N}$, and let $\Gamma$ be one of its faces, say $\left\{\left(x_{1}, \ldots, x_{N}\right): 0 \leq x_{i} \leq L\right.$ for $1 \leq i \leq N-1$; and $\left.x_{N}=L\right\}$. We have the following result:

Proposition 3.1 Let $\lambda_{k}^{\left(Q_{L}\right)}$ and $\Lambda_{k}^{\left(Q_{L}\right)}$ be the $k^{\text {th }}$-eigenvalues of Problem 1.1 and Problem 2.2 in $Q_{L}$. Then,

$$
\Lambda_{k}^{\left(Q_{L}\right)}>\lambda_{k}^{\left(Q_{L}\right)}
$$

Moreover, when $k \geq 2$,

$$
\lambda_{k}^{\left(Q_{L}\right)} \sim \lambda_{k}^{\left(Q_{1}\right)} L^{1-p}
$$

Proof. Let us recall the variational characterization of eigenvalues,

$$
\begin{aligned}
& \lambda_{k}^{\left(Q_{L}\right)}=\inf _{F \in C_{k}^{Q_{L}}} \sup _{u \in F} \frac{\int_{Q_{L}}|\nabla u|^{p}+|u|^{p}}{\int_{\partial Q_{L}}|u|^{p}}, \\
& \Lambda_{k}^{\left(Q_{L}\right)}=\inf _{F \in C_{k}^{Q_{L}}} \sup _{u \in F} \frac{\int_{Q_{L}}|\nabla u|^{p}+|u|^{p}}{\int_{\Gamma}|u|^{p}},
\end{aligned}
$$

where

$$
C_{k}^{Q_{L}}=\left\{C \subset W^{1, p}\left(Q_{L}\right): C \text { compact, } C=-C, \gamma(C) \geq k\right\} .
$$

Since $\Gamma \subset \partial \Omega$, the inequality $\Lambda_{k}^{\left(Q_{L}\right)}>\lambda_{k}^{\left(Q_{L}\right)}$ holds.

Now, the proof that $\lambda_{k}^{\left(Q_{L}\right)} \sim \lambda_{k}^{\left(Q_{1}\right)} L^{1-p}$ can be found in [9], and follows by changing variables in the Rayleigh quotient, since $|\nabla u|^{p}$ contributes with the factor $L^{-p}$, and the differentials of volume and surface area differs by a factor $L$.

Remark 3.1 Let us note that for $k=1$ this is not true, since $\lambda_{k}^{\left(Q_{L}\right)}$ goes to zero when $L$ decreases. See [9] for further details on scaling.

Our next Theorem gives the upper bound for the eigenvalue counting function for certain domains.

Theorem 3.1 Let us suppose that $\Gamma_{1}$ is an hyperplane. Then,

$$
N_{s N}\left(\lambda, \Omega, \Gamma_{1}\right) \leq \frac{\left|\Gamma_{1}\right|_{N-1} \lambda^{\frac{N-1}{p-1}}}{\left(\lambda_{2}^{\left(Q_{1}\right)}\right)^{N-1}} .
$$

Proof. Let $\lambda$ be fixed, and let us divide $\Omega$ in several parts: cubes $\left\{Q_{L}^{i}\right\}_{1 \leq i \leq J}$ with one of its faces in $\Gamma_{1}$, and a complementary set $Q^{c}=\Omega \backslash \cup_{i=1}^{J}{\overline{Q_{L}}}^{i}$.

Now, applying the Dirichlet Neumann Bracketing by decomposing $\Omega$ as $Q^{c} \cup\left(\cup_{i=1}^{J} Q_{L}^{i}\right)$, with the eigenvalue parameter only on the faces of the cubes in $\Gamma_{1}$ and zero Neumann boundary conditions in the others and in $\partial Q^{c}$ gives an upper bound for $N_{s N}\left(\lambda, \Omega, \Gamma_{1}\right)$ given by

$$
\sum_{i=1}^{J} N\left(\lambda, Q_{L}^{i}\right)
$$


We are free to choose the length $L$ yet. By using Proposition 3.1, if $L<\left(\lambda_{2}^{\left(Q_{1}\right)} / \lambda\right)^{1 /(p-1)}$, then $\Lambda_{2}^{\left(Q_{L}\right)}>\lambda$, and therefore $N\left(\lambda, Q_{L}^{i}\right) \leq 1$.

Hence, we choose $L=\left(\lambda_{2}^{\left(Q_{1}\right)} / \lambda\right)^{1 /(p-1)}$ and we can bound $N\left(\lambda, \Omega, \Gamma_{1}\right)$ by the number of cubes $J$, and by equating surface areas we obtain $J L^{N-1}=\left|\Gamma_{1}\right|_{N-1}$, that is,

$$
N_{s N}\left(\lambda, \Omega, \Gamma_{1}\right) \leq \frac{\left|\Gamma_{1}\right|_{N-1} \lambda^{\frac{N-1}{p-1}}}{\left(\lambda_{2}^{\left(Q_{1}\right)}\right)^{N-1}}
$$

and the proof is finished.

\section{A Lower Bound for $N(\lambda)$}

We will consider now the semi-Dirichlet eigenvalue problem:

$$
\left\{\begin{aligned}
\Delta_{p} u & =|u|^{p-2} u & & \text { in } \Omega \\
|\nabla u|^{p-2} \frac{\partial u}{\partial \nu} & =\Lambda|u|^{p-2} u & & \text { on } \Gamma \\
u & =0 & & \text { on } \partial \Omega \backslash \Gamma .
\end{aligned}\right.
$$

We consider the particular case $\Omega=R_{L}=[0, L]^{N-1} \times[0, L / 2]$, and let $\Gamma$ be the face $\left\{\left(x_{1}, \ldots, x_{N}\right): 0 \leq x_{i} \leq L\right.$ for $1 \leq i \leq N-1$; and $\left.x_{N}=L / 2\right\}$. We obtain upper bounds for $\Lambda_{1}^{\left(R_{L}\right)}$ from the previous bounds of $\mu_{1}^{\left(R_{L}\right)}$ in Proposition 2.1. We have the following result:

Proposition 4.1 Let $\Lambda_{1}^{\left(R_{L}\right)}$ and $\mu_{1}^{\left(R_{L}\right)}$ be the first eigenvalues of Problems 2.1 and 2.7 in $R_{L}$. Then,

$$
\Lambda_{1}^{\left(R_{L}\right)} \leq\left(\mu_{1}^{\left(R_{L}\right)}+1\right) \frac{L}{2} .
$$

Proof. Let us recall the variational characterization of the first eigenvalue,

$$
\Lambda_{1}^{\left(R_{L}\right)}=\inf _{u \in W^{1, p}\left(Q_{L}\right),\left.u\right|_{\partial R_{L} \backslash \Gamma}=0} \frac{\int_{R_{L}}|\nabla u|^{p}+|u|^{p}}{\int_{\Gamma}|u|^{p}} .
$$

Now, we use as a test function the first eigenfunction $\varphi$ of problem 2.7, obtaining

$$
\Lambda_{1}^{\left(R_{L}\right)} \leq\left(\frac{\int_{R_{L}}|\nabla \varphi|^{p}+|\varphi|^{p}}{\int_{R_{L}}|\varphi|^{p}}\right)\left(\frac{\int_{R_{L}}|\varphi|^{p}}{\int_{\Gamma}|\varphi|^{p}}\right) \leq\left(\mu_{1}^{\left(R_{L}\right)}+1\right)\left(\frac{\int_{R_{L}}|\varphi|^{p}}{\int_{\Gamma}|\varphi|^{p}}\right) .
$$

Finally, by using Fubini's Theorem, the second factor could be bounded by $L / 2$ and the proof is complete.

Remark 4.1 Combining the previous Proposition with Proposition 2.1, we have

$$
\Lambda_{1}^{\left(R_{L}\right)} \leq \frac{C_{p}}{2}\left(\frac{\pi_{p}^{p} N}{L^{p-1}}+L\right) \sim \frac{\pi_{p}^{p} C_{p} N}{2 L^{p-1}}
$$

when $L \rightarrow 0$. 
As in the previous section, the next Theorem gives the lower bound for the eigenvalue counting function in certain domains.

Theorem 4.1 Let $N_{s D}\left(\lambda, \Omega, \Gamma_{1}\right)$ be the spectral counting function of Problem 2.1 where $\Gamma_{1}$ is an hyperplane. Then,

$$
N_{s D}\left(\lambda, \Omega, \Gamma_{1}\right) \geq c\left|\Gamma_{1}\right|_{N-1} \lambda^{\frac{N-1}{p-1}},
$$

where $c$ depends only on $p$ and $N$.

Proof. Let $\lambda$ be fixed, and let us divide $\Omega$ in several parts as before: rectangles $\left\{R_{L}^{i}\right\}_{1 \leq i \leq J}$ with one of this faces in $\Gamma$, and a complementary set $R^{c}=\Omega \backslash \cup_{i=1}^{J}{\overline{R_{L}}}^{i}$.

Now, applying the Dirichlet Neumann bracketing by decomposing $\Omega$ as $R^{c} \cup_{i=1}^{J} R_{L}^{i}$, with the eigenvalue parameter only on the faces of the rectangles in $\Gamma_{1}$ and zero Dirichlet boundary conditions in the others and in $\partial R^{c}$ gives an upper bound for $N_{s D}\left(\lambda, \Omega, \Gamma_{1}\right)$ given by

$$
\sum_{i=1}^{J} N\left(\lambda, R_{L}^{i}\right) .
$$

We choose now the length $L$. By using the remark below Proposition 2.1, if $L=$ $\left(\pi_{p}^{p} C_{p} N / 2 \lambda\right)^{1 /(p-1)}$, then each rectangle has at least one eigenvalue, and therefore $N\left(\lambda, R_{L}^{i}\right) \geq$ 1 .

Hence, $N_{s D}\left(\lambda, \Omega, \Gamma_{1}\right)$ is greater than or equal to $J$, and by equating surface areas we obtain $J L^{N-1}=\left|\Gamma_{1}\right|_{N-1}$, that is,

$$
N_{s D}\left(\lambda, \Omega, \Gamma_{1}\right) \geq c\left|\Gamma_{1}\right|_{N-1} \lambda^{\frac{N-1}{p-1}}
$$

\section{Proof of Theorem 1.1}

The proof of Theorem 1.1 is rather lengthy and technical, and follows the same lines of sections 4.3 - 4.5 in [18]. We only sketch the main steps, since the critical estimations were given in Theorems 3.1 and 4.1.

Given an arbitrary open set $\Omega$ with smooth boundary, we divide its boundary in a finite number of connected parts $\left\{S_{j}\right\}_{1 \leq j \leq J}$, and there exist $J$ coordinate neighborhoods $U_{j} \subset$ $\mathbb{R}^{N-1}, \varphi_{j}$ such that any $x \in S_{j}$ is given by the equation $x=\varphi\left(y_{1}, \ldots, y_{N-1}\right)$.

Now, given $x \in S_{j}$, let $\nu_{x}$ be the outer unit normal at $x$, and let us introduce the sets

$$
D_{j}=\left\{z: z=x-y_{N} \nu_{x}, x \in S_{j}, 0<y_{N}<\delta\right\} .
$$

Clearly, $\delta$ could be chosen such that $D_{j} \subset \Omega$ for $1 \leq j \leq J$.

The first step in the proof of Theorem 1.1 is the following Lemma, which is a consequence of the Dirichlet Neumann bracketing: 
Lemma 5.1 Let $D_{j}$ and $S_{j}$ be defined as before. Then,

$$
\sum_{j=1}^{J} N_{s D}\left(\lambda, D_{j}, S_{j}\right) \leq N(\lambda, \Omega, \partial \Omega) \leq \sum_{j=1}^{J} N_{s N}\left(\lambda, D_{j}, S_{j}\right)
$$

Let $\varepsilon>0$ be fixed, and let us introduce the set $\varphi_{j}^{-1}\left(D_{j}\right)=V_{j} \times(0, \delta)$, where $V_{j} \subset U_{j}$. By a change of variables, the eigenvalue problem could be studied on the sets $V_{j} \subset U_{j}$. By subdividing $S_{j}$ and after an orthogonal transformation if necessary, the jacobian of the transformation could be bounded as $1 \pm \varepsilon$ in the variational characterization of eigenvalues.

Hence, $\left|S_{j}\right|_{N-1} \sim\left|V_{j}\right|_{N-1}$, and we have (as in subsections 4.5.4 and 4.5.5 of [18]), the next step of the proof:

Lemma 5.2 Let $D_{j}, S_{j}$ and $V_{j} \times(0, \delta)$ be defined as before. Then,

$$
\begin{aligned}
& N_{s N}\left(\lambda, D_{j}, S_{j}\right) \sim N_{s N}\left(\lambda, V_{j} \times(0, \delta), V_{j} \times\{0\}\right), \\
& N_{s D}\left(\lambda, D_{j}, S_{j}\right) \sim N_{s D}\left(\lambda, V_{j} \times(0, \delta), V_{j} \times\{0\}\right) .
\end{aligned}
$$

Let us note that the asymptotic expansion of $N_{s N}\left(\lambda, V_{j} \times(0, \delta), V_{j} \times\{0\}\right)$ and $N_{s D}\left(\lambda, V_{j} \times\right.$ $\left.(0, \delta), V_{j} \times\{0\}\right)$ was obtained in Theorems 3.1 and 4.1 respectively, and therefore we have the last step of the proof:

Lemma 5.3 Let $S_{j}$ and $V_{j} \times(0, \delta)$ be defined as before. Then,

$$
\begin{aligned}
& N_{s N}\left(\lambda, V_{j} \times(0, \delta), V_{j} \times\{0\}\right) \leq C\left|S_{j}\right|_{N-1} \lambda^{\frac{N-1}{p-1}} \\
& N_{s D}\left(\lambda, V_{j} \times(0, \delta), V_{j} \times\{0\}\right) \geq c\left|S_{j}\right|_{N-1} \lambda^{\frac{N-1}{p-1}} .
\end{aligned}
$$
$N(\lambda)$.

Combining this result with Lemma 5.1, we obtain the desired asymptotic bounds for

Remark 5.1 Let us note that Corollary 1.1 follows immediately from Theorem 1.1 since $N\left(\lambda_{k}\right)=k$ gives the inequalities

$$
c|\partial \Omega|_{N-1} \lambda_{k}^{\frac{N-1}{p-1}} \leq k \leq C|\partial \Omega|_{N-1} \lambda_{k}^{\frac{N-1}{p-1}} .
$$

\section{References}

[1] G. Bognar and O. Dosly, The Application of Picone-type Identity for some Nonlinear Elliptic Differential Equations, Acta Math. Univ. Comenian. (NS) LXXII ,1, (2003), 45 57.

[2] R. Courant and D. Hilbert, Methods of Mathematical Physics, Vol 1, Interscience Publishers, Inc., New York, 1953.

[3] P. Drabek and R. Manásevich, On the Closed Solutions to some nonhomegeneous eigenvalue problemes with p-laplacian, Diff. Int. Equations, 12, 6, (1999), 773-788. 
[4] J. Fernández Bonder and J. P. Pinasco, Asymptotic behavior of the eigenvalues of the onedimensional wheighted p-Laplace operator, Arkiv fur Mathematik 41 (2003) 267-280.

[5] J. Fernández Bonder and J. P. Pinasco, Estimates for Eigenvalues of Quasilinear Elliptic Systems, Part II, Preprint (2006).

[6] J. Fernández Bonder, J. P. Pinasco, and J. D. Rossi, Asymptotic of eigenvalues of the Steklov $\infty$-Laplace eigenvalues, in preparation.

[7] J. Fernández Bonder and J. D. Rossi, Existence results for the p-Laplacian with nonlinear boundary conditions, J. Math. Anal. Appl. 263 (2001), 195-223.

[8] J. Fernández Bonder and J. D. Rossi, A nonlinear eigenvalue problem with indefinite weights related to the Sobolev trace embedding, Publ. Mat. 46 (2002), 221-235.

[9] J. Fernández Bonder and J. D. Rossi. Asymptotic behavior of the best Sobolev trace constant in expanding and contracting domains, Comm. Pure Appl. Anal. 1, 3 (2002), 359378.

[10] J. Fleckinger, E. M. Harrell II, and F. de Thélin, Boundary behavior and estimates for solutions of equations containing the p-laplacian, Electron. J. Differential Equations 1999, 38 (1999), 1-20.

[11] L. Friedlander, Asymptotic behaviour of eigenvalues of the p-Laplacian Commun. Part. Diff. Eqs. 14, 8/9, (1989) 1059-1069.

[12] J. García Azorero and I. Peral Alonso, Comportement asymptotique des valeurs propres du p-laplacien, C. R. Acad. Sci. Paris 307, Serie I, (1988) 75-78.

[13] Hormander, L. The Analysis of linear partial differential operators, Vol. III, Springer Verlag, 1985.

[14] M. Kac, Can one hear the shape of a drum?, Amer. Math. Monthly (Slaught Mem. Papers, 11 4, 73, (1966) 1-23

[15] S. Martinez and J. D. Rossi, Isolation and simplicity for the first eigenvalue of the plaplacian with a nonlinear boundary condition, Abstr. Appl. Anal. 7, 5, (2002) 287-293.

[16] J. P. Pinasco, The Asymptotic behavior of Nonlinear Eigenvalues. To appear in Rocky Mountain J. of Math.

[17] P. Rabinowitz, Minimax methods in critical point theory with applications to differential equations, CBMS Reg. Conf. Ser. Math., no. 65, Amer. Math. Soc., Prov., R.I. 1986.

[18] L. Sandgren, A vibration problem, Medd. Lund Univ. Mat. Sem., 13 (1955), 1-84. 